\title{
Embryonic Zebrafish: Different Phenotypes after Injection of Human Uveal Melanoma Cells
}

\author{
Wietske van der Ent ${ }^{a, b} \quad$ Claudia Burrello ${ }^{a}$ Mark J. de Lange ${ }^{c}$ \\ Pieter A. van der Velden ${ }^{c}$ Aart G. Jochemsen ${ }^{d}$ Martine J. Jager ${ }^{c}$ \\ B. Ewa Snaar-Jagalska ${ }^{a}$ \\ ${ }^{a}$ Institute of Biology, Leiden University, and Departments of ${ }^{b}$ Pathology, ${ }^{c}$ Ophthalmology \\ and ${ }^{\mathrm{d}}$ Molecular Cell Biology, Leiden University Medical Center, Leiden, The Netherlands
}

Key Words
Uveal melanoma $\cdot$ Zebrafish $\cdot$ Drug screening $\cdot$ Crizotinib

\begin{abstract}
Although murine xenograft models for human uveal melanoma (UM) are available, they are of limited utility for screening large compound libraries for the discovery of new drugs. We need new preclinical models which can efficiently evaluate drugs that can treat UM metastases. The zebrafish embryonic model is ideal for drug screening purposes because it allows the investigation of potential antitumor properties of drugs within 1 week. The optical transparency of the zebrafish provides unique possibilities for live imaging of fluorescence-labelled cancer cells and their behavior. In addition, the adaptive immune response, which is responsible for the rejection of transplanted material, is not yet present in the early stages of fish development, and systemic immunosuppression is therefore not required to allow growth of tumor cells. We studied the behavior of UM cells following injection into zebrafish embryos and observed different phenotypes. We also analyzed cell migration, proliferation, formation of micrometastasis and interaction with the host microenvironment. Significant differences were noted between cell lines: cells derived from metastases showed more migration and proliferation than cells derived from the primary tumors. The addition of the c-Met inhibitor crizotinib to the water in which the larvae were kept reduced the migration and proliferation of UM cells expressing c-Met. This indicates the applicability of the zebrafish xenografts for testing novel inhibitory compounds and provides a fast and sensitive in vivo vertebrate model for preclinical drug screening to combat UM.




\section{Ocular Oncology \\ and Pathology}

van der Ent et al.: Embryonic Zebrafish: Different Phenotypes after Injection of Human Uveal Melanoma Cells

\section{Introduction}

New drugs are often identified on the basis of in vitro analyses. However, even if drug development proceeds smoothly at the early in vitro stages, it may fail during the in vivo preclinical stages. This may be due to the toxicity of the compound, which causes unexpected side effects. According to estimates by the Federal Drug Administration (FDA), a 10\% improvement in predicting drug failures prior to clinical trials could save USD 100 million in development costs per drug [1]. It would therefore be useful to find better drug screening systems. An alternative could be the use of in vivo drug discovery systems with good clinical observations of animals. Aside from murine transgenic or syngeneic implantation models, murine xenograft models are being used, but the use of zebrafish (Danio rerio) is another option. Zebrafish screening is quick, inexpensive, consumes small amounts of compounds and does not require much space [2,3]. Because of these reasons, the zebrafish has recently become popular as an experimental biological model. It is highly suitable for toxicity screenings, and, indeed, studies have shown that many toxic responses are well conserved between fish and mammals [4].

Of course, there are still obstacles to overcome before the zebrafish is recognized as a model for drug discovery $[3,4]$. First of all, it is not known yet how the zebrafish absorbs the chemical compounds dissolved in the egg water. The zebrafish embryo is thought to take up the compounds by diffusion through the skin and the gills. It is also possible that the embryo ingests the compound through the gastrointestinal tract. Certainly, the rate of absorbance depends on the physicochemical properties of the drug. To properly understand the toxicity of a compound, bioanalyses are needed to measure the body burden of a drug in the embryo. Due to the small dimensions of the embryo, tissue-specific bioanalyses are difficult to perform. However, the application of radio-labelled compounds has allowed precise studies of different body areas. Comparisons of bioanalyses of the head and trunk area have shown that some drugs can cross the blood-brain barrier, while others cannot [5]. This shows that, with regard to this barrier, zebrafish resemble the rodent model.

Bioanalysis can also be performed on the fluid that remains in the well at the end of the assay, providing further information on the pharmacokinetics of the compound. If the concentration of the compound is significantly reduced at the end of the experiment, this suggests that the drug has been readily taken up by the embryo. Additionally, the disappearance of the drug can be compared to the body burden. If the body burden is smaller than the decrease of the compound, the compound has been metabolized by the embryo [6]. This may be important as a rapid metabolism may lead to reduced activity and detoxification. On the other hand, the production of active metabolites could cause an increase in the toxicity.

Zebrafish have many useful properties, such as a large clutch size (the adult fish can produce up to 200 offspring per week), ex utero development, easy manipulation of the embryo and optical transparency. Zebrafish embryos are particularly suitable for microscopic imaging, because they are translucent and can be maintained in small wells for long periods [7]. They can be observed by stereomicroscopy, and individual organs can be monitored. With increasing magnification and resolution, one can see structures at the cellular level. This allows, for instance, the monitoring of the interaction between the innate immune system and cancer cells [8]. Specifically focusing on cancer, zebrafish models have the advantage that they have highly conserved oncogenes and tumor suppressor genes that they share with humans [9]. This has permitted the use of zebrafish in several cancer models, for instance in leukemia [10-14]. Interactions between cancer cells and the immune system can be studied as embryos already possess a functional innate immune system 1 day after development [15]. Functional neutrophils are present by day 2 . In addition, the adaptive immune response, which is responsible for the rejection of transplanted material, is not present yet in 
the early stages of development; thus, immunosuppression of the animal is not required. This lack of a specific immune response allows xenotransplantation in early-stage embryos [16].

We set out to develop a uveal melanoma (UM) experimental metastasis model; such a model may be used to screen potential anti-UM drugs, to study interactions between the immune system and UM cells in vivo, or to compare the migratory characteristics of different UM. We have recently reported that the zebrafish UM model that we developed can be used efficiently to test the efficiency of new anti-UM drugs [17]. However, one can inject tumor cells into zebrafish through several routes, which may lead to different types of tumor growth; those different phenotypes will be discussed in the following section.

\section{Setting Up a UM Model in Zebrafish}

Zebrafish and embryos were raised, staged and maintained according to standard procedures in compliance with Dutch animal welfare regulations. Cell lines were grown in RPMI 1640 medium and Dulbecco's modified Eagle's medium in a 1:1 ratio with $15 \%$ fetal calf serum. Cell lines Mel270, OMM2.3 and OMM2.5 were a gift from Dr. B.R. Ksander [18], cell line 92.1 came from our own laboratory [19] and cell line OMM1 was a gift from Dr. G.P.M. Luyten [20]. All cell lines were stably transduced with CMV-mCherry and selected with puromycin for the stable expression of red fluorescence.

The zebrafish embryonic model is ideal for xenograft studies as it allows the investigation of tumorigenic properties of cancer cells within 1 week, with unique possibilities for live imaging of fluorescence-labelled cancer cells because of the zebrafish optical transparency [21]. For an optimal visualization, the transgenic zebrafish line Tg(fli1:EGFP) expressing a green fluorescent vasculature was used [22]. One of the models used yolk sac implantation and has recently been described extensively [17]. The technique is as follows: before implantation, eggs were kept at $28^{\circ} \mathrm{C}$ and manually dechorionated at 1 day post-fertilization (dpf). Cells were grown in vitro and suspended in $7 \mu \mathrm{l}$ of $2 \%$ polyvinylpyrrolidone-40 (PVP-40; Calbiochem) and injected using glass capillary needles ( $1 \mathrm{~mm}$ OD $\times 0.78 \mathrm{~mm}$ ID; Harvard Apparatus) with an approximate opening equal to the dimension of 1 cell. Circa 400-500 cells stably expressing mCherry were injected into the yolk sac of 2-dpf embryos using a Pneumatic Pico pump and a manipulator (World Precision Instruments). After the implantation, embryos were kept in egg water $(60 \mu \mathrm{g} / \mathrm{ml}$ OceanSalt in demineralized water $)$ at $34^{\circ} \mathrm{C}$. The embryos were screened at 1 day post-injection (dpi) using a stereo fluorescence microscope, and embryos with low amounts of engrafted cells or with a faint glowing fluorescence throughout the yolk (indicative of mechanical damaging of the cells) were removed. Per cell line, 150 embryos were injected, of which more than $90 \%$ were correctly engrafted. The survival rate of the injected embryos at $6 \mathrm{dpi}$ was $60-70 \%$. Using the stereo fluorescence microscope, embryos were imaged alive at 4, 5 and 6 dpi to observe the development of the different phenotypes. At 6 dpi, embryos were fixed overnight in $4 \%$ paraformaldehyde (PFA) for subsequent confocal imaging and automated analysis. Figure 1 depicts an overview of the workflow described above.

\section{Cancer Cells Engrafted in the Yolk Sac of Zebrafish Embryos Undergo Active Migration}

At $4 \mathrm{dpi}$, the cancer cells were mostly scattered along the whole yolk sac. Simultaneously, migration of cells outside the yolk sac was observed. Cancer cell dissemination was considered active migration if the cells had moved beyond the boundaries of the heart cavity frontally, on top of the swim bladder dorsally and farther than the urogenital opening caudally. 
Ocular Oncology

and Pathology

Fig. 1. Workflow of the zebrafish embryonic xenograft model. Selected UM cell lines stably expressing mCherry were implanted into the yolk of 2-dpf TG(fli1:EGFP) embryos with green fluorescent vessels. These embryos are selected at $1 \mathrm{dpi}$ for correct engraftment in the yolk. Embryos with little to no cells in the yolk or cells in the bloodstream are removed from the experiment. Using stereomicroscopy at 4, 5 and $6 \mathrm{dpi}$, the embryos are checked for migration and proliferation of engrafted cells. At 6 dpi, embryos were fixed overnight with $4 \% \mathrm{PFA}$ at $4{ }^{\circ} \mathrm{C}$. Fixed embryos were used for confocal fluorescence microscopy and automated quantitative analysis of tumor burden.

\begin{tabular}{l|l}
\hline Ocul Oncol Pathol 2015;1:170-181 \\
\hline DOI: $10.1159 / 000370159$ & $\begin{array}{l}\text { @ 2015 S. Karger AG, Basel } \\
\text { www.karger.com/oop }\end{array}$ \\
\hline
\end{tabular}

van der Ent et al.: Embryonic Zebrafish: Different Phenotypes after Injection of Human Uveal Melanoma Cells

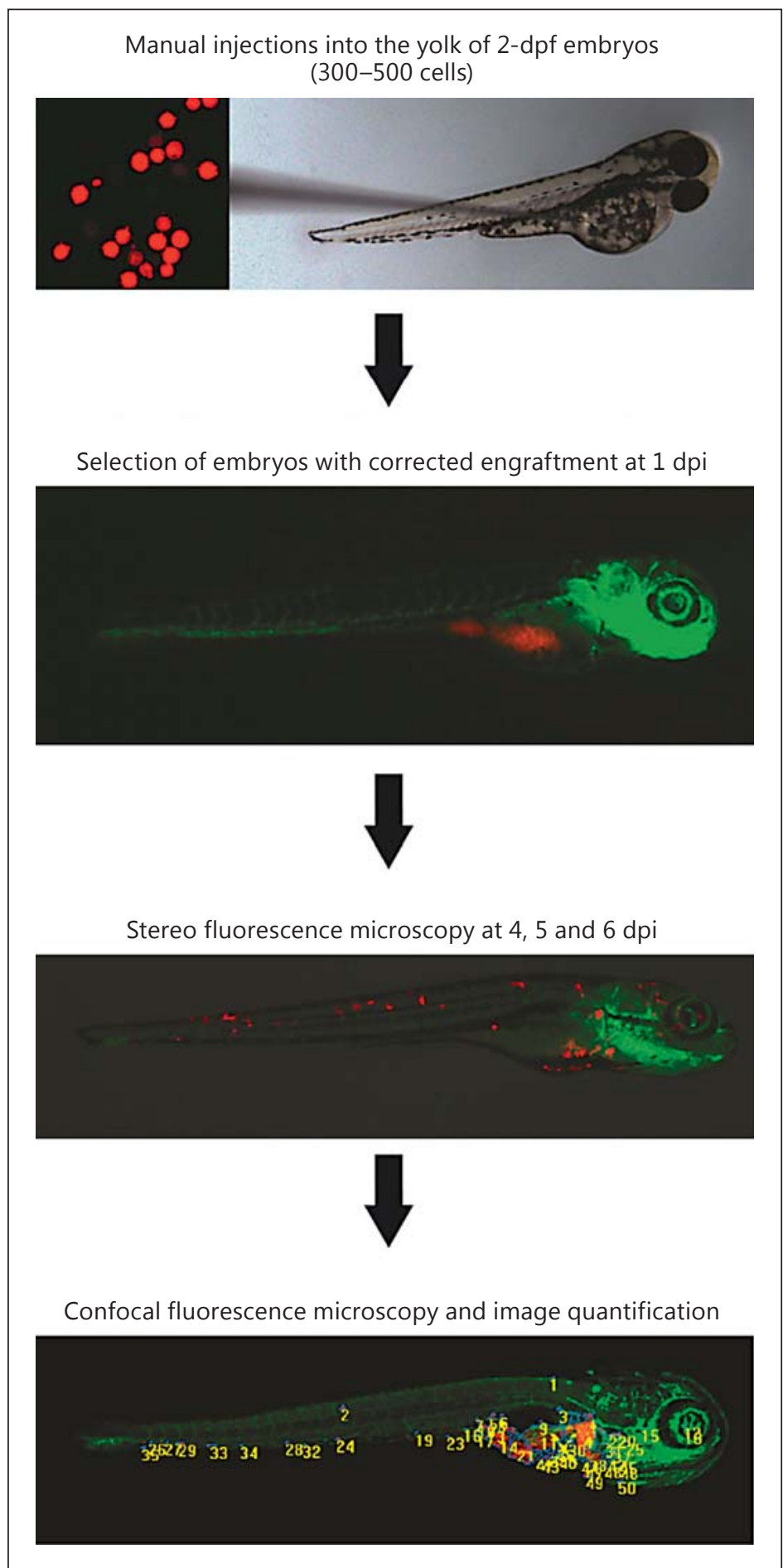

Migration occurred both in the head and in the tail region. In both cases, there were cancer cells which, according to the 2-dimensional stereo images, seemed to be inside the vasculature. At 5 and $6 \mathrm{dpi}$, the percentage of embryos with migrating cells increased to approximately $50 \%$ of the total amount of embryos injected. Figure 2 shows UM cells in the yolk sac right after implantation (fig. 2a) and migrating cells to the tail (Mel270, fig. 2b) or the tail and the head (OMM2.3, fig. 2c) at $6 \mathrm{dpi}$. 
Ocular Oncology

and Pathology

Fig. 2. Examples of UM cell phenotypes at 1 and $6 \mathrm{dpi}$ and the method of quantification. a Representative image of a 2-day-old Tg(fli1:EGFP) embryo with mCherry-expressing UM cells engrafted in the yolk. b A $\mathrm{Tg}$ (fli1:EGFP) embryo with Mel270 cells migrating to the tail at $6 \mathrm{dpi}$. c A $\mathrm{Tg}$ (fli1:EGFP) embryo with OMM2.3 cells migrating to the tail at $6 \mathrm{dpi}$. Reproduced with permission from Investigative Ophthalmology and Visual Sciences [17].

\begin{tabular}{l|l}
\hline Ocul Oncol Pathol 2015;1:170-181 \\
\hline DOI: $10.1159 / 000370159$ & $\begin{array}{l}\text { (c) 2015 S. Karger AG, Basel } \\
\text { www.karger.com/oop }\end{array}$ \\
\hline
\end{tabular}

van der Ent et al.: Embryonic Zebrafish: Different Phenotypes after Injection of Human Uveal Melanoma Cells
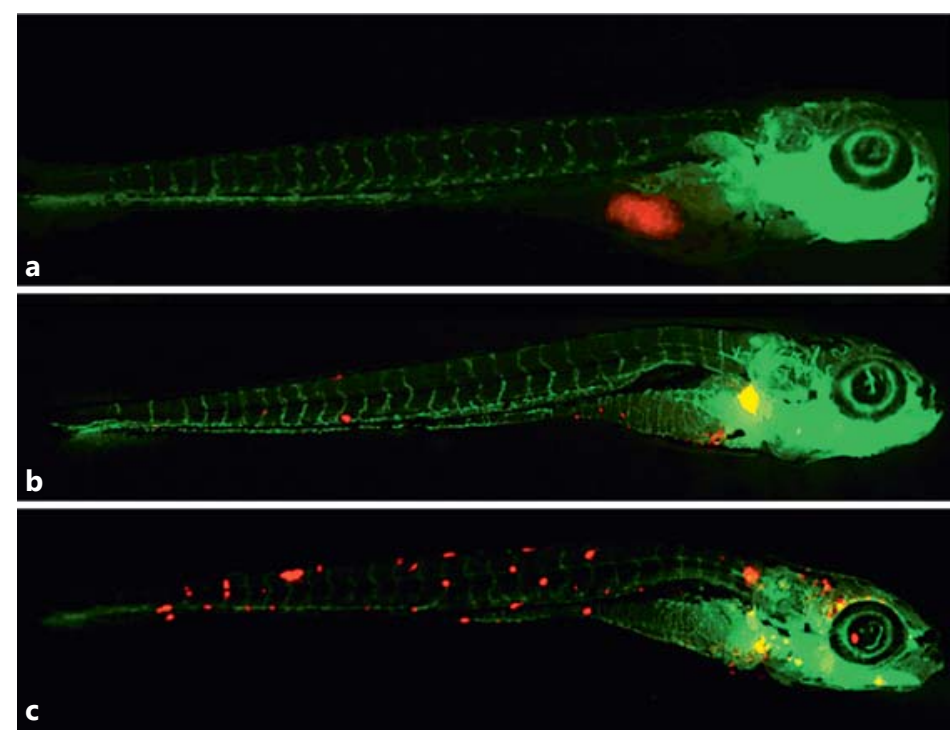

\section{In the Zebrafish Embryonic Xenograft Model, Each UM Cell Line Has a Different Invasiveness}

Clinical experience indicates that nowadays every cancer patient needs a personalized treatment strategy because even patients affected by the same type of cancer can respond differently to the same treatment. One can obtain cells from each malignancy to determine its individual sensitivity to drugs. Different cell lines have been isolated and stabilized or immortalized in order to be able to culture and, therefore, study them. We used several human UM cell lines, which have different origins and molecular features.

In order to establish a biological research model to study UM, it was fundamental to describe how these different cell lines behave in zebrafish embryos in an objective manner. To do so, an automated whole-animal bioimaging assay was used. For this analysis, 6-dpi embryos were fixed with 4\% PFA and imaged with low-magnification confocal laser scanning microscopy. Projections of these z-stack images were analyzed using an Image-Pro Analyzer macro, developed in order to obtain a quantitative measurement of cell proliferation and migration [23]. This macro is able to detect every red fluorescent cluster of cells inside the embryo and quantify its dimension and its distance to the injection point (fig. 3). To determine the cancer cell burden per embryo, the mean area of all red tumor cell clusters is multiplied by the total number of red objects (area $\times$ objects). To quantify migration, the distance between each cell cluster and the site of implantation (SOI) is measured and averaged for all the clusters (mean migration) or added up (cumulative/total migration) within 1 embryo.

Figure 4 displays the result of the quantitative analysis of the tumor burden (fig. $4 \mathrm{a}$ ) and migration (fig. 4b) for five UM cell lines. Mel270, which is a cell line derived from a primary tumor, clearly proliferates and migrates less compared to OMM2.3 and OMM2.5, which were isolated from two liver metastases found in the same patient. The tumor burden induced by Mel270 was significantly lower than the burden induced by a different primary tumor cell line, i.e. 92.1. Furthermore, the metastatic OMM1 cell line migrated more than Mel270, while its proliferation was only slightly but not significantly higher compared to that of Mel270.

In addition to the quantifiable UM cell behavior in the zebrafish embryo host described in the paragraph above, additional phenotypes were observed between 4 and 6 dpi. Some embryos injected with UM cells showed a mass protruding out of the yolk that composed of 


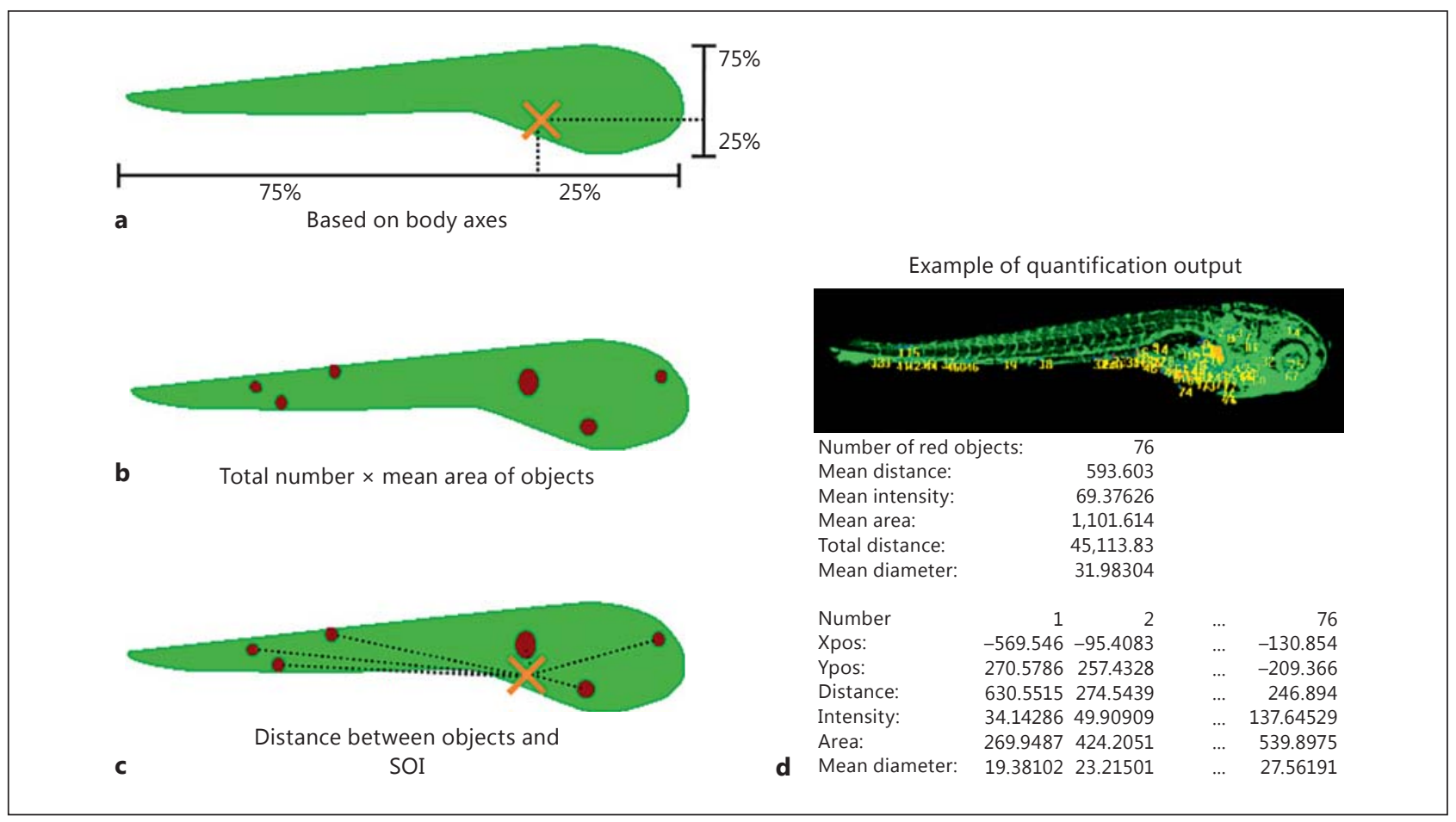

Fig. 3. Scheme explaining automated quantitative image analysis. a The SOI (orange X) is determined based on the body axes of the embryo. The green fluorescent vasculature of the $\mathrm{Tg}$ (fli1:EGFP) embryo allows the localization of these axes, and the SOI is mapped at 25\% of the horizontal axis (from the caudal side) and at $25 \%$ of the vertical axis (from the ventral side). b Based on the red fluorescent signal, the number of cell clusters is determined. This number is multiplied by the mean area of all the clusters present in the embryo to quantify the total tumor burden per embryo. c For the quantification of migration, the distance between each cluster of tumor cells and the SOI is determined and averaged (mean distance) or added up (total/cumulative distance). $\mathbf{d}$ Example of the output of automated quantitative analysis.

red fluorescent UM cells and nonfluorescent zebrafish stromal cells (fig. 5a). This phenotype was present with a low incidence (1-2\%) and was not visible at $1 \mathrm{dpi}$, suggesting it is not related to the mechanical process of the injection. These outgrowing tumors were stable: when present at $4 \mathrm{dpi}$, they remained intact until $6 \mathrm{dpi}$.

In order to investigate the interaction of migrating cells with the adaptive immune cells of the zebrafish larvae, an L-plastin immunohistochemical staining was performed (fig. 5b). In those embryos, where neutrophils and macrophages were stained blue, no interaction between UM cells and L-plastin-positive cells was detected.

Another particularly interesting phenotype was that approximately $10 \%$ of the embryos injected showed the presence of cancer cells in 1 or both eyes. This phenotype was observed for both Mel270 (fig. 5c) and OMM2.3 (fig. 5d) cell lines.

\section{Effect of Crizotinib on UM Xenografts}

We used the yolk sac model to determine the effect of drugs on migration and proliferation [17]. We tested the effect of crizotinib, a multitarget tyrosine kinase inhibitor, to evaluate c-Met inhibition. OMM2.3, which showed the most aggressive phenotype in the zebrafish 
Fig. 4. Characterization of in vivo behavior of 5 UM cell lines in the zebrafish embryonic xenograft model. a Quantification of tumor burden plotted on a logarithmic scale. Symbols indicate the tumor burden per embryo, calculated multiplying the mean area (determined from fluorescent pixels) of all identified foci by the number of red objects detected. b Mean distance of migration away from the SOI per red object in a single embryo, plotted on a linear scale. The data presented are the results of two repeated experiments for each cell line. Statistical significance was calculated by one-way analysis of variance with Dunnett's post-test, and p values were indicated as follows: $* * \mathrm{p}<0.01$. For all groups: $\mathrm{n} \geq 39$. Reproduced with permission from Investigative Ophthalmology and Visual Science [17].

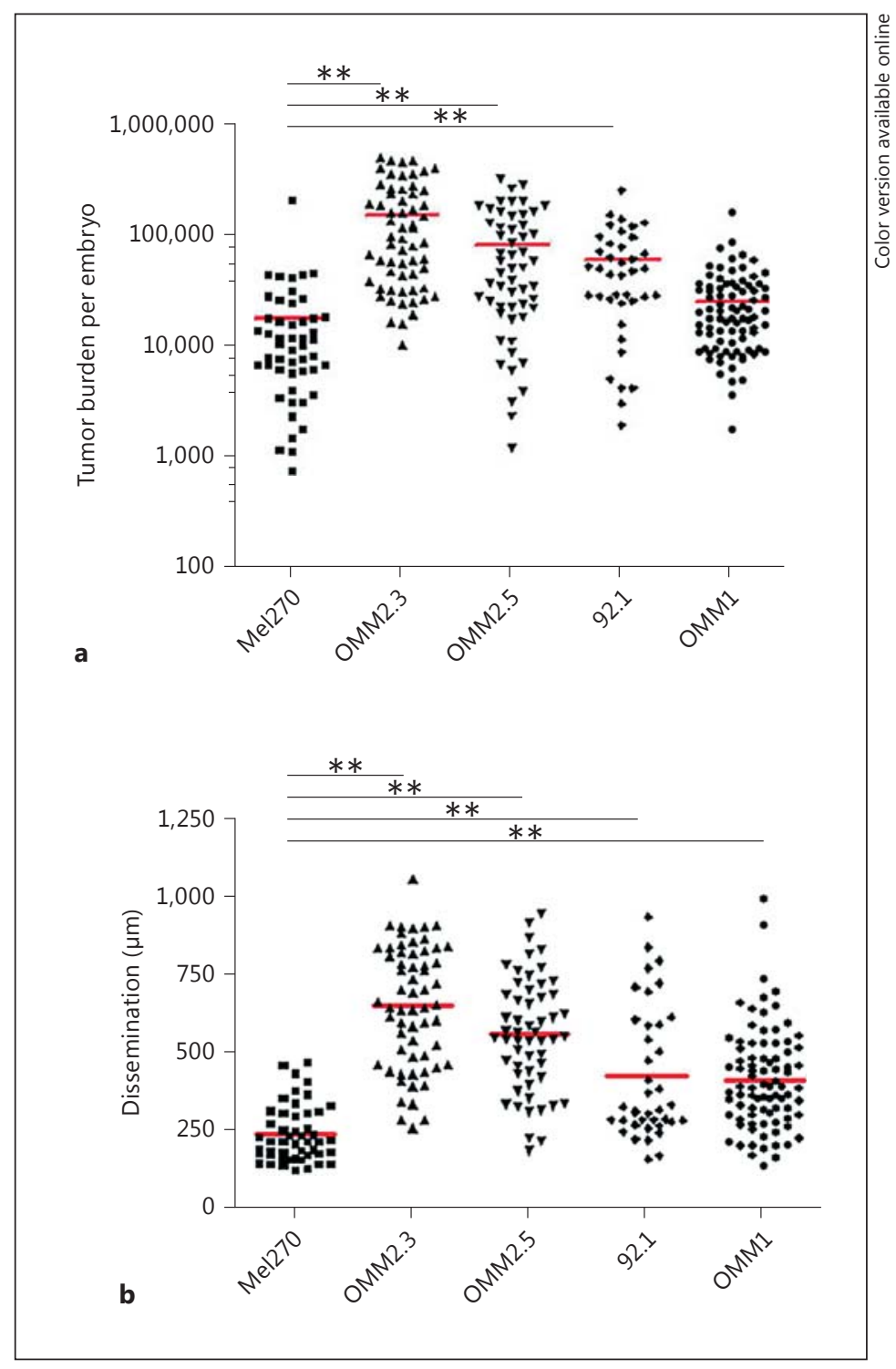

embryonic xenograft model, has an overactivation of c-MET. The treatment of this cell line with crizotinib was expected to reduce the malignant phenotype. In addition to OMM2.3, the primary UM cell line 92.1 was treated with crizotinib. In this cell line, c-MET is only modestly expressed and is not activated. Therefore, crizotinib was expected to have hardly any effect on this cell line.

Embryos injected with either OMM2.3 or 92.1 cells at $2 \mathrm{dpf}$ were treated from $1 \mathrm{dpi}$ onwards with $5 \mu \mathrm{M}$ crizotinib. This compound was refreshed every day, and at $6 \mathrm{dpi}$, the embryos were fixed and imaged for automated quantification of tumor cell burden and migration. Figure 6 shows the effect of crizotinib on OMM2.3 and 92.1 in the zebrafish embryonic xenograft model. Both the tumor cell burden and migration of OMM2.3 were significantly reduced after treatment with crizotinib. In contrast, a similar treatment of 92.1 showed no reduction in tumor cell burden nor in migration of these cells. These results confirm that the model is applicable to determine the differential effects of various drugs on UM cells in vivo in a time-effective manner. 
Ocular Oncology

and Pathology
Ocul Oncol Pathol 2015;1:170-181

DOI: $10.1159 / 000370159$

van der Ent et al.: Embryonic Zebrafish: Different Phenotypes after Injection of Human Uveal Melanoma Cells
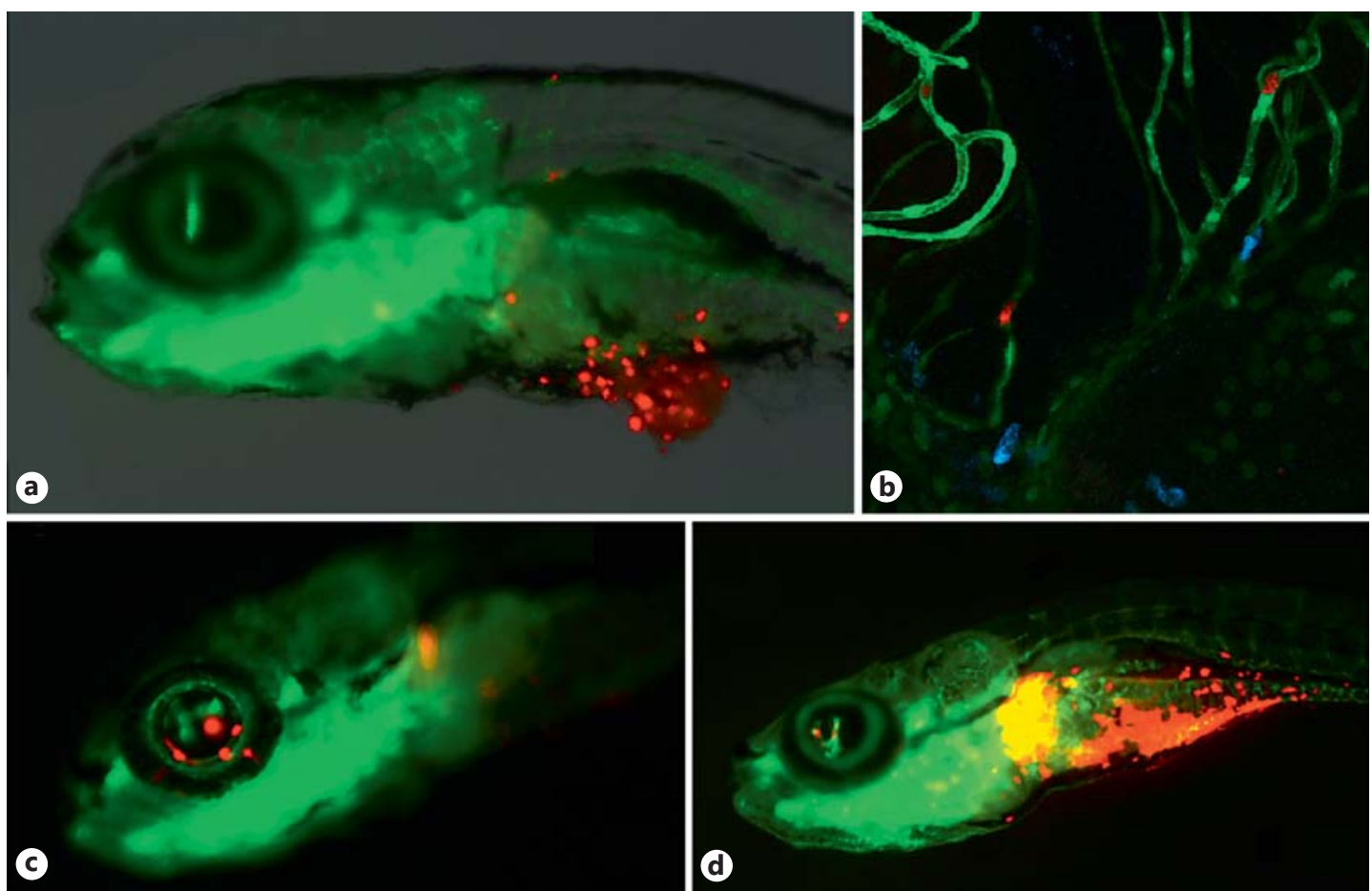

Fig. 5. Phenotypes observed between 4 and 6 dpi. a A Tg(fli1:EGFP) embryo with an outgrowth of engrafted Mel270 cells (red) from the yolk at $4 \mathrm{dpi}$. b Detail confocal image of a Tg(fli1:EGFP) embryo showing OMM2.3 cells (red) within the vasculature (green) at $6 \mathrm{dpi}$. L-plastin-positive cells (blue) did not interact with the UM cells. c A Tg(fli1:EGFP) embryo with Mel270 cells present in the eye at 4 dpi. d A Tg(fli1:EGFP) embryo with OMM2.5 cells which show proliferation in the yolk and migrate towards the head and eye of the embryo at $4 \mathrm{dpi}$.

\section{Injections into the Blood Circulation Stimulate the Formation of UM Micrometastases}

In order to additionally establish an experimental UM metastasis model in vivo, tumor cells were injected directly into the circulation of 2-dpf zebrafish embryos. As a result, cancer cells passively disseminated immediately after the implantation. Therefore, cells did not need to intravasate in order to migrate, as was necessary for the injections into the yolk. Cells were manually injected into the duct of Cuvier, a large blood vessel that surrounds the embryonic yolk sac connecting the heart to the trunk vasculature. This method has already widely been used to study micrometastasis formation in zebrafish embryonic xenograft models [24, 25]. Alternatively, cells were injected directly into the heart cavity.

One day after implantation into the bloodstream, UM cells had spread throughout the body but were still present in the blood circulation. At 6 dpi, embryos were fixed with 4\% PFA and imaged with a Leica SPE Confocal Microscope for a more in-depth analysis. At this point, melanoma cells had extravasated and were found in the extracellular matrix surrounding the vessels. Their elongated morphology indicated a possible interaction with the new microenvironment and suggests motility of these cells.

After direct injection of UM cells into the blood circulation, the embryos very frequently exhibited micrometastases in the eye: at $5 \mathrm{dpi}$, this phenotype was found in more than $80 \%$ of the embryos. Kalirai et al. [26] describe the same phenomenon for the chick embryo. To further analyze the characteristics of this process, live confocal imaging was performed at 
Fig. 6. In vivo effect of crizotinib on OMM2.3 and 92.1 cells. Effect of $5 \mu \mathrm{M}$ crizotinib on tumor cell burden (a) and migration (b) of OMM2.3 and 92.1 cells. Statistical significance was calculated by Student's t test, and p values were indicated as follows: ${ }^{* *} \mathrm{p}<0.01$; $* * * \mathrm{p}<0.001$. For all groups: $\mathrm{n} \geq 30$.

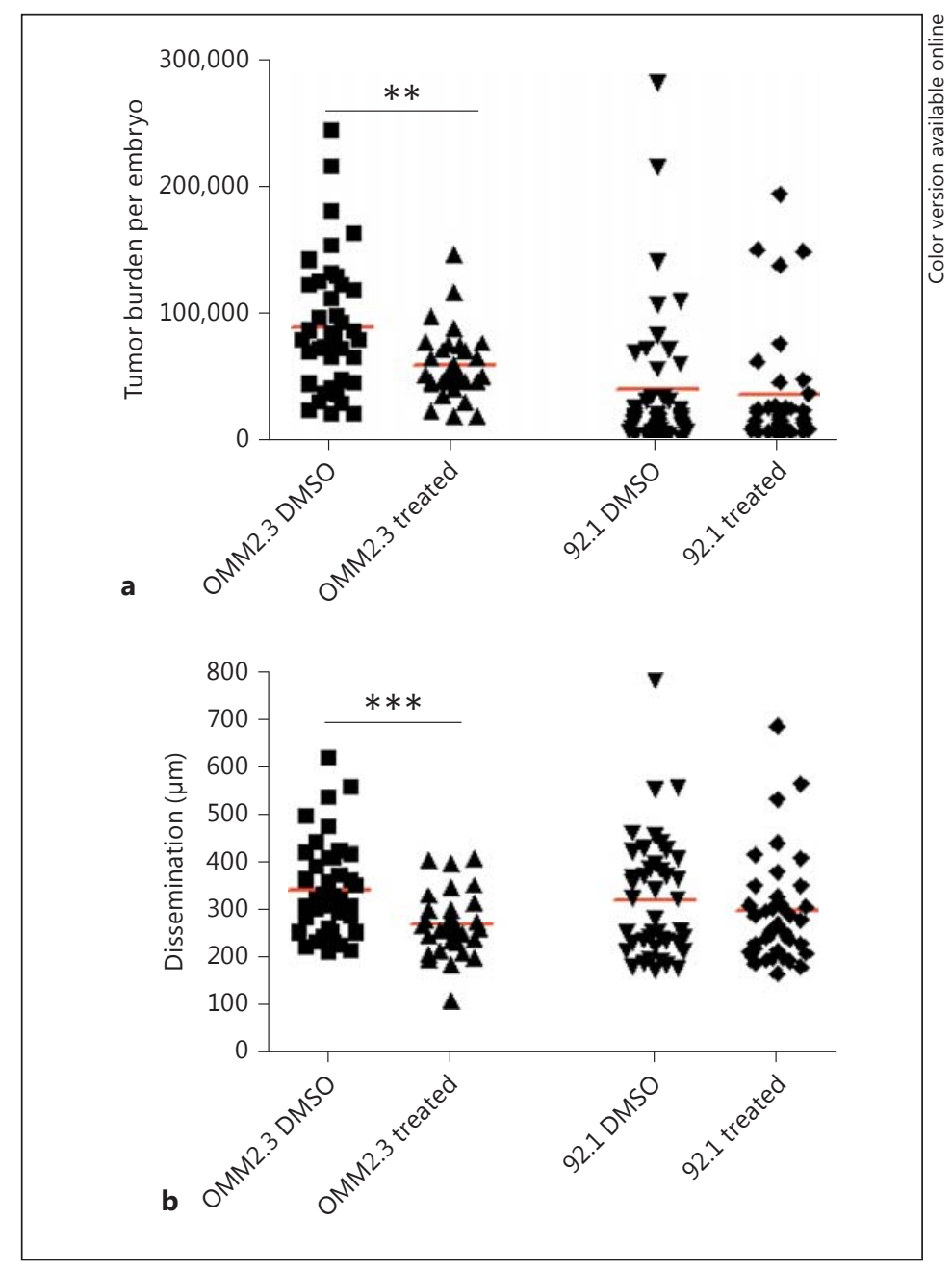

multiple time points in zebrafish embryos that had been injected with OMM1/mCherry cells into the heart cavity. Figure $7 \mathrm{a}$ and $\mathrm{b}$ shows the same eye of an embryo imaged at 3 and $4 \mathrm{dpi}$, respectively. At $3 \mathrm{dpi}$, the melanoma cells were mainly inside the vasculature, but they were clearly interacting with the vessel wall. One day later, the tumor load inside the eye has considerably increased, the mCherry signal has expanded, and a mass of cells was seen outside the vasculature. Given the fact that, at this stage, no more circulating melanoma cells are visible when watching the embryos under the stereo fluorescence microscope, one can hypothesize that the UM cells are proliferating at the site of the metastasis. Figure $7 \mathrm{c}-\mathrm{f}$ shows OMM1 cells injected into the heart cavity at 6 dpi, fixed and stained with Ki67. Positive Ki67 staining here indicates that these cell are proliferating at this time point.

\section{Conclusion}

Patients presenting with metastatic UM have a poor prognosis as effective therapeutic strategies are currently lacking [27]. For these patients, it is critical that novel treatment regimens can progress rapidly from bench to bedside. To aid this process, the testing of new potential anticancer drugs in vivo is essential. A variety of xenotransplantation models in 
Ocular Oncology

and Pathology

van der Ent et al.: Embryonic Zebrafish: Different Phenotypes after Injection of Human Uveal Melanoma Cells
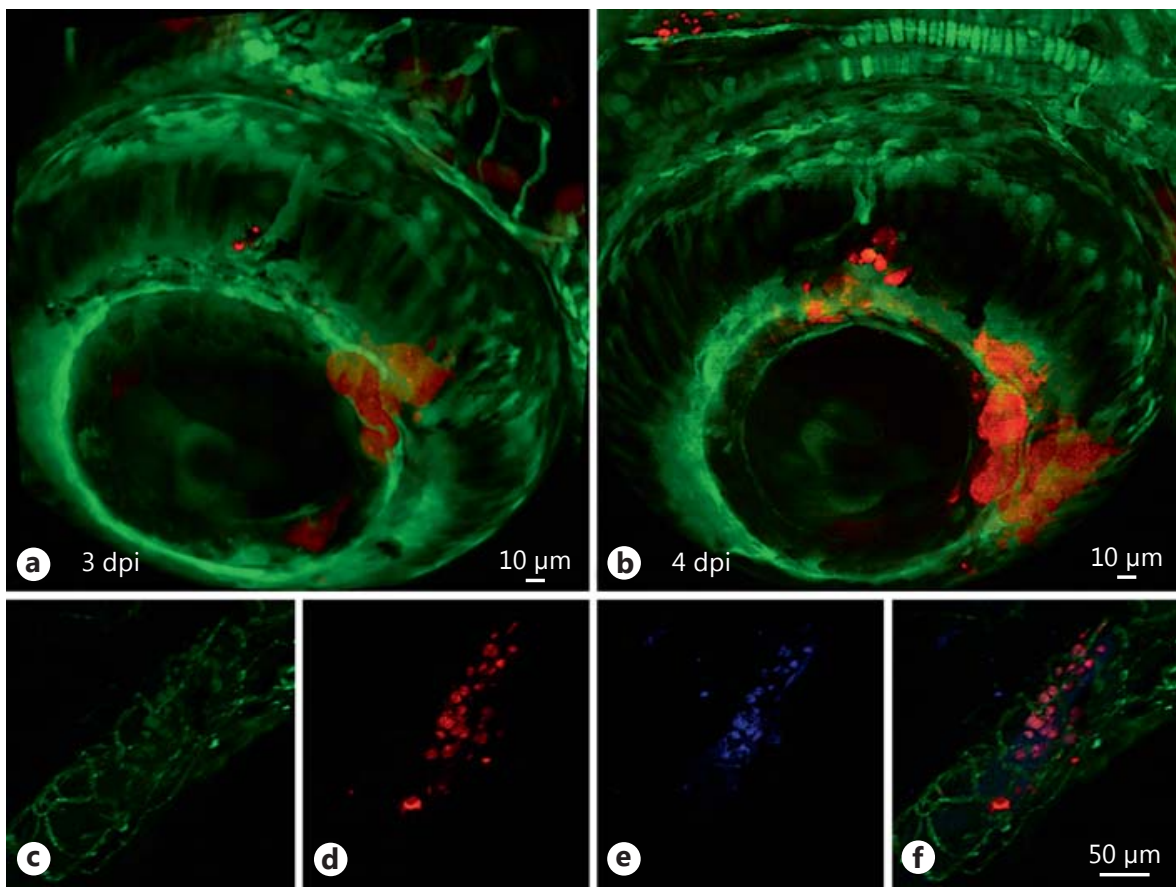

Fig. 7. Proliferation of UM metastases in embryos injected into the heart cavity. a Eye of a Tg(fli1:EGFP) embryo injected with OMM1 cells (red) into the heart cavity at $3 \mathrm{dpi}$. Cells show interaction with the blood vessel wall (green) ( $\times 20$ objective and $1.8 \mathrm{zoom}$ ). b Image of the eye shown in a at $4 \mathrm{dpi}$. OMM1 cells have extravasated, and the tumor load is increased ( $\times 20$ objective and 1.8 zoom). c-f A 6-dpi embryo injected with OMM1 cells (red) into the heart cavity. Images were taken at the yolk sac area and show the proliferation of these cells ( $\times 10$ objective). c Green signal of TG(fli1:EGFP) vasculature. $\mathbf{d}$ Red signal from OMM1 mCherry cells. e Blue signal showing Ki67-positive cells. $\mathbf{f}$ Merged channel image of $\mathbf{c}, \mathbf{d}$ and $\mathbf{e}$.

immunodeficient mice has been developed, where UM cells are either engrafted within the anterior chamber or vitreous cavity of the eyeball, or directly into the liver, representing the common site of metastatic spread for these tumors [28-34]. Although these mouse models provide valuable information on the response of UM to drugs in vivo, they have several drawbacks. The tumor growth/spread in these models is too slow. Furthermore, the high variation in the tumor growth rate requires a large cohort of mice to test drug efficacy, which is costly and not desirable for large-scale drug screens.

In this paper, we discussed zebrafish as a useful in vivo model to aid in drug discovery for the treatment of UM. We showed that UM cells engrafted into the yolk of a 2-day-old zebrafish embryo are able to proliferate and migrate throughout the host. This proliferation and spread could be abated by adding a c-Met inhibitor, crizotinib, to the water in which the larvae were maintained. The ability of zebrafish larvae to take up compounds from the water in which they are kept is an especially attractive feature with regard to drug screens. At the time of the described xenograft experiments, zebrafish larvae were sufficiently small to be kept in 96-well plates, reducing the amount of compound needed for these tests.

In addition to the migration and proliferation of engrafted UM cells, other phenotypes were observed. In some embryos, solid masses of cancer and zebrafish cells formed outgrowths from the abdomen of the larvae. In other embryos, engrafted cells were seen to form micrometastases in the eye. This phenotype was greatly increased when cells were engrafted directly into the bloodstream via the heart cavity or the duct of Cuvier. Further investigation 
into this phenotype showed that the cells seeding at this location were interacting directly with the blood vessels present and were proliferating with time.

In summary, this paper highlights the potential that the zebrafish xenograft model has to aid in preclinical analysis of new anticancer compounds against UM in a time- and costeffective manner.

\section{Acknowledgments}

This work was supported by Stichting Kinderen Kankervrij, Project 30677, and by Dutch Cancer Society grant UL 2011-4991.

\section{References}

1 US Food and Drug Administration: Innovation or stagnation? Challenge and opportunity on the critical path to new medical products. 2004. http://www.fda.gov/ScienceResearch/SpecialTopics/CriticalPathInitiative/ CriticalPathOpportunitiesReports/ucm077262.htm.

-2 Zon LI, Peterson RT: In vivo drug discovery in the zebrafish. Nat Rev Drug Discov 2005;4:35-44.

3 Santoriello C, Zon LI: Hooked! Modeling human disease in zebrafish. J Clin Invest 2012;122:2337-2343.

-4 Peterson RT, Macrae CA: Systematic approaches to toxicology in the zebrafish. Annu Rev Pharmacol Toxicol 2012;52:433-453.

5 Fleming A, Diekmann H, Goldsmith P: Functional characterisation of the maturation of the blood-brain barrier in larval zebrafish. PLoS One 2013;8:e77548.

-6 Alderton W, Berghmans S, Butler P, Chassaing H, Fleming A, Golder Z, Richards F, Gardner I: Accumulation and metabolism of drugs and CYP probe substrates in zebrafish larvae. Xenobiotica 2010;40:547-557.

7 Ignatius MS, Langenau DM: Fluorescent imaging of cancer in zebrafish. Methods Cell Biol 2011;105:437-459.

-8 Feng Y, Renshaw S, Martin P: Live imaging of tumor initiation in zebrafish larvae reveals a trophic role for leukocyte-derived $\mathrm{PGE}_{2}$. Curr Biol 2012;22:1253-1259.

-9 Howe K, Clark MD, Torroja CF, et al: The zebrafish reference genome sequence and its relationship to the human genome. Nature 2013;496:498-503.

10 Mione MC, Trede NS: The zebrafish as a model for cancer. Dis Model Mech 2010;3:517-523.

11 Goessling W, North TE, Zon LI: New waves of discovery: modeling cancer in zebrafish. J Clin Oncol 2007;25: 2473-2479.

12 Konantz M, Balci TB, Hartwig UF, Dellaire G, Andre MC, Berman JN, Lengerke C: Zebrafish xenografts as a tool for in vivo studies on human cancer. Ann NY Acad Sci 2012;1266:124-137.

13 Amatruda JF, Shepard JL, Stern HM, Zon LI: Zebrafish as a cancer model system. Cancer Cell 2002;1:229-231.

14 White R, Rose K, Zon L: Zebrafish cancer: the state of the art and the path forward. Nat Rev Cancer 2013;13: 624-636.

15 Lieschke GJ, Trede NS: Fish immunology. Curr Biol 2009;19:R678-R682.

16 Stoletov K, Klemke R: Catch of the day: zebrafish as a human cancer model. Oncogene 2008;27:4509-4520.

-17 van der Ent W, Burrello C, Teunisse AF, Ksander BR, Van der Velden PA, Jager MJ, Jochemsen AG, SnaarJagalska BE: Modeling of human uveal melanoma in zebrafish xenograft embryos. Invest Ophthalmol Vis Sci 2014;55:6612-6622.

18 Chen PW, Murray TG, Uno T, Salgaller ML, Reddy R, Ksander BR: Expression of MAGE genes in ocular melanoma during progression from primary to metastatic disease. Clin Exp Metastasis 1997;15:509-518.

19 De Waard-Siebinga I, Blom DJ, Griffioen M, Schrier PI, Hoogendoorn E, Beverstock G, Danen EH, Jager MJ: Establishment and characterization of an uveal-melanoma cell line. Int J Cancer 1995;62:155-161.

-20 Luyten GP, Naus NC, Mooy CM, Hagemeijer A, Kan-Mitchell J, Van Drunen E, Vuzevski V, De Jong PT, Luider TM: Establishment and characterization of primary and metastatic uveal melanoma cell lines. Int J Cancer 1996; 66:380-387.

-21 van der Ent W, Jochemsen AG, Teunisse AF, Krens SG, Szuhai K, Spaink HP, Hogendoorn PC, Snaar-Jagalska BE: Ewing sarcoma inhibition by disruption of EWSR1-FLI1 transcriptional activity and reactivation of p53. J Pathol 2014;233:415-424.

22 Lawson ND, Weinstein BM: In vivo imaging of embryonic vascular development using transgenic zebrafish. Dev Biol 2002;248:307-318.

23 Ghotra VP, He S, de Bont H, van der Ent W, Spaink HP, van de Water B, Snaar-Jagalska BE, Danen EH: Automated whole animal bio-imaging assay for human cancer dissemination. PLoS One 2012;7:e31281.

24 He S, Lamers GE, Beenakker JW, Cui C, Ghotra VP, Danen EH, Meijer AH, Spaink HP, Snaar-Jagalska BE: Neutrophil-mediated experimental metastasis is enhanced by VEGFR inhibition in a zebrafish xenograft model. J Pathol 2012;227:431-445. 
Drabsch Y, He S, Zhang L, Snaar-Jagalska BE, Ten Dijke P: Transforming growth factor-beta signalling controls human breast cancer metastasis in a zebrafish xenograft model. Breast Cancer Res 2013;15:R106.

26 Kalirai H, Shahidipour H, Coupland SE, Luyten GPM: Use of the chick embryo model in uveal melanoma. Ocul Oncol Pathol 2015;1:133-140.

27 Field MG, Harbour JW: GNAQ/11 mutations in uveal melanoma: is YAP the key to targeted therapy? Cancer Cell 2014;25:714-715.

28 Cao J, Jager MJ: Animal eye models for uveal melanoma. Ocul Oncol Pathol 2015;1:141-150.

29 Yang H, Cao J, Grossniklaus HE: Uveal melanoma metastasis models. Ocul Oncol Pathol 2015;1:151-160.

-30 Braun RD, Vistisen KS: Modeling human choroidal melanoma xenograft growth in immunocompromised rodents to assess treatment efficacy. Invest Ophthalmol Vis Sci 2012;53:2693-2701.

-31 Heegaard S, Spang-Thomsen M, Prause JU: Establishment and characterization of human uveal malignant melanoma xenografts in nude mice. Melanoma Res 2003;13:247-251.

-32 Kan-Mitchell J, Mitchell MS, Rao N, Liggett PE: Characterization of uveal melanoma cell lines that grow as xenografts in rabbit eyes. Invest Ophthalmol Vis Sci 1989;30:829-834.

33 Nemati F, Sastre-Garau X, Laurent C, Couturier J, Mariani P, Desjardins L, Piperno-Neumann S, Lantz O, Asselain B, Plancher C, Robert D, Peguillet I, Donnadieu MH, Dahmani A, Bessard MA, Gentien D, Reyes C, Saule S, Barillot E, Roman-Roman S, Decaudin D: Establishment and characterization of a panel of human uveal melanoma xenografts derived from primary and/or metastatic tumors. Clin Cancer Res 2010;16:2352-2362.

-34 Yang H, Fang G, Huang X, Yu J, Hsieh CL, Grossniklaus HE: In-vivo xenograft murine human uveal melanoma model develops hepatic micrometastases. Melanoma Res 2008;18:95-103. 